\title{
Desempeño académico y diferencias de género en Colombia: un análisis con base en las pruebas TIMSS 2007'
}

\author{
Academic Performance and Gender \\ Differences in Colombia: An Analysis Based \\ on the TIMMS 2007 Examination Results
}

\section{Desempenho acadêmico e diferenças de gênero na Colombia: uma análise com base em provas TIMSS 2007}

Juan Byron Correa Fonnegra²

Docente e investigador de la Universidad del Valle, Cali, Colombia

juan.correa@correounivalle.edu.co

Recibido: 21/08/2014

Aprobado: 24/11/2015

1 El proyecto de investigación "Brecha escolar de género en Colombia: un estudio aplicado con base en las pruebas PIRLS, TIMSS y PISA" fue financiado por la Vicerrectoría de investigaciones de la Universidad del Valle en la convocatoria interna 2012, código CI 6150. El autor agradece la valiosa colaboración del estudiante de Maestría en Economía Aplicada, Carlos Andrés Orejuela y de la estudiante de Economía, Ángela María Rodríguez, miembros del Grupo de investigación en Economía Regional y Ambiental (GERA) del cual es miembo. 


\title{
Resumen
}

Este artículo analiza las características que inciden en el desempeño de los estudiantes colombianos en el área de matemáticas. Con base en la información de la prueba TIMSS 2007, evalúa el impacto sobre las brechas en el desempeño entre hombres y mujeres en los grados cuarto y octavo. El estudio evidencia que, además de las condiciones innatas, las características que inciden en mayor proporción sobre el desempeño académico son, en primer lugar, el entorno familiar del estudiante, que en buena parte determina el comportamiento individual y su interacción dentro de la sociedad, y segundo, las creencias y/o confianza que el estudiante tiene en su capacidad y su esfuerzo en las actividades matemáticas.

Palabras claves: diferencias de género; autoconfianza; efecto de los pares; efectos endógenos; prueba TIMSS.

\begin{abstract}
This article analyses the characteristics of the academic performance of Colombian students in mathematics. Based on information from the TIMSS 2007 test evaluates the impact on performance gaps between men and women in the fourth and eighth grades. The study reveals that in addition to innate conditions, there are two characteristics that affects mostly on academic performance: First, the student's family environment, which largely determines individual behavior and his interaction within society; second, the beliefs and the confidence that the student has in his ability and his effort in mathematics activities.
\end{abstract}

Keywords: gender differences; self-confidence; peer effect; endogenous effects; TIMSS.

\section{Resumo}

Este artigo analisa as características que incidem no desempenho dos estudantes colombianos na área de matemáticas. A partir da informação da prova TIMSS 2007, avalia-se o impacto sobre as brechas no desempenho entre homens e mulheres de quarto e oitavo grau do ensino médio nacional. O estudo evidencia que, além das condições inatas, as características que mais incidem sobre o desempenho académico são, em primeiro lugar, o entorno familiar do estudante que em grande medida determina o comportamento individual e sua interação dentro da sociedade; e em segundo lugar, as crenças y/o desconfiança que tem o estudante sobre sua capacidade e esforço nas atividades matemáticas.

Palavras-chave: diferenças de gênero; autoconfiança; efeito dos pares; efeitos endógenos; prova TIMSS.

(c) (i) Este trabajo está bajo la licencia Creative Commons Attribution 3.0

¿Cómo citar este artículo? / How to quote this article?

Correa, Juan «Desempeño académico y diferencias de género en Colombia: un análisis con base en las pruebas TIMSS 2007». Sociedad y economía, No. 30 (enero - junio 2016): 15-42. 


\section{Introducción}

Está bien documentado que las habilidades y capacidades son las características que en mayor proporción inciden en el desempeño académico de los estudiantes; pero dado que tanto habilidades como capacidades varían de alumno a alumno, es de esperar que el rendimiento igualmente varíe, resultado que se refleja en los diferenciales en cuanto a género, tipo de institución, ubicación del colegio o nivel social y económico del hogar al que pertenece el estudiante.

¿Qué explica las diferencias en las habilidades y capacidades de los estudiantes? La literatura referencia el papel fundamental de la familia en la formación de las habilidades y capacidades de los hijos, así como las influencias genéticas; pero dado que la formación de habilidades es un proceso dinámico que comienza en la etapa de gestación y se multiplica en cada una de las etapas subsiguientes, un mecanismo de inversiones tempranas por parte de los padres podría reducir las diferencias en las habilidades entre los niños de diferente condición socioeconómica.

El ambiente en el que se desarrolla el estudiante también es parte fundamental del proceso de formación de habilidades y por ende en su desempeño. La interacción constante con los padres, compañeros de clase y el vecindario, las actividades extracurriculares en que participa y en general las diferentes formas de interacción social permiten que el estudiante, dependiendo del entorno social, mejore sus habilidades y capacidades innatas más adecuada y eficientemente.

Por otro lado, aunque el tamaño de la clase es un factor muy importante en el proceso de enseñanza, los estudios indican que la composición del aula es fundamental. Un alto grado de homogeneidad en el salón de clase permite un proceso de interacción más ágil entre los estudiantes; las afinidades sociales y culturales de los alumnos en el aula los hacen más propensos a comportarse de forma similar a la predominante en el grupo, es decir, conductas similares dentro de los grupos facilitan la presencia de efectos endógenos dentro del salón de clase. Coleman et al. (1966) y Arnott y Rowse (1987) afirman que las capacidades de los compañeros de clase son más importantes en el desempeño escolar individual que las capacidades innatas del estudiante.

El impacto de pertenecer a un grupo de referencia específico, como lo resalta Manski (1993), puede generar efectos diferenciales (endógenos, contextuales o correlacionados); tanto el entorno familiar como el no familiar en los que se desenvuelve el alumno son primordiales en este proceso. El desempeño individual de los estudiantes puede estar influenciado por el logro promedio del grupo de referencia al que pertenece. Así mismo, una característica exógena del grupo de referencia puede incidir directamente sobre el desempeño individual. Por último, en ciertos entornos, dado que los estudiantes presentan similares capitales sociales y culturales, tienden a presentar desempeños muy homogéneos, producto de la constante interacción en ambientes similares.

El desempeño del estudiante en matemáticas no solo depende de sus condiciones innatas, sino que también está influenciado por el entorno del hogar así como por la interacción dentro y fuera del salón de clase.

Para explicar la brecha entre niños y niñas en la prueba de matemáticas, Bandura (1993), con base en la definición de autoeficacia, utiliza la valoración que 
tiene el estudiante del grado de confianza en sí mismo de resolver un problema matemático, concepto denominado autoeficacia percibida.

Las creencias y valoraciones que un estudiante tiene de sí mismo cumplen una función relevante en el procesamiento de la información y en la regulación de la conducta; la autoeficacia es la creencia en las propias capacidades para organizar y ejecutar los cursos de acción necesarios para gestionar situaciones posibles (Pajares 1996). Al hablar de autoeficacia se hace referencia a una autoevaluación específica del contexto de competencia para llevar a cabo una tarea; una alta percepción de eficacia mejora el rendimiento de la memoria ya que motiva a la persistencia, además influencia el logro académico directa e indirectamente, elevando la calidad de los objetivos que se propone el estudiante.

Este estudio utiliza un enfoque psicosocial para explicar las diferencias en el desempeño en matemáticas entre hombres y mujeres, utilizando un conjunto de variables proxy de los procesos de socialización de los estudiantes tanto en el hogar como en la institución, y una medida del nivel de autocreencias en el desempeño en la prueba por parte del estudiante. Es usual encontrar estudios sobre evaluación educativa en una u otra dirección, pero, lo cierto es que existe una alta correlación entre ambos componentes que motiva un estudio conjunto.

En el presente artículo, se presenta una revisión de literatura tanto internacional como nacional; se describen el modelo analítico, los datos y la metodología utilizada, y se presenta el análisis tanto descriptivo como de la estimación del modelo multinivel donde se destacan los hallazgos más importantes. En la última sección se relacionan las conclusiones del estudio, así como algunas propuestas de política; además se hace una relación de los principales problemas u omisiones en el estudio.

\section{Literatura relacionada}

El desempeño académico se ha constituido en un campo de investigación no solo de las ciencias sociales sino también de la psicología y la educación misma. Las diversas corrientes teóricas desarrolladas respecto al campo educativo plantean importantes postulados, por ejemplo, la posición frente a la desigualdad social y el papel de las instituciones educativas modernas en el proceso de formación, planteada por la nueva sociología de la educación. Por otro lado, la necesidad de acercarse más al modo en que los factores personales, tanto cognitivos como no cognitivos, interactúan con el contexto de la clase durante el proceso de aprendizaje constituyen importantes temas de investigación para los psicólogos educacionales (Bandura 1982 y 1993). Así mismo, la economía de la educación -apoyada en sus dos teorías principales que relacionan el nivel de estudios de un individuo con el nivel de ingresos que obtendrá a lo largo de su ciclo vital一, la teoría del capital humano -introducida a finales de los años cincuenta por Schultz (1961) y modelada más tarde con rigor por Becker ([1964] 1994) - y la teoría de la señalización — propuesta por economistas y sociólogos como Spence (1973), Arrow (1973) y Stiglitz (1975) - dejan en evidencia la importancia de alcanzar una educación de calidad. 
El informe Equality of Educational Opportunity de Coleman et al. (1966), ampliamente conocido por cuestionar el papel del sistema educativo estadounidense en la generación de igualdad de oportunidades para la movilidad social, destaca entre sus conclusiones que las brechas en los logros académicos entre los estudiantes de diferentes condiciones raciales no son causadas per se por las instituciones, sino que influye más el medio en el que vive el estudiante que la igualdad en los elementos físicos de la escuela, la calidad de su currículo o el nivel de formación de los profesores. Posteriormente, Arnott y Rowse (1987) consolidan los anteriores resultados al afirmar que además de las capacidades innatas del estudiante, las capacidades promedio de los compañeros de clase son determinantes en el desempeño académico individual, influencia denominada efecto compañeros de grupo o "efecto pares".

En la década de 1970, Bernstein (1975) citado en Giddens (2001), en la teoría de los códigos, resaltó los diferentes códigos o formas de discurso que desarrollan los niños según su procedencia social. A partir del análisis de las diferencias en el uso del lenguaje entre niños ricos y pobres encuentra que los niños de clase trabajadora tendrían un código restringido, familiar y de vecindad, vinculado a su entorno cultural más inmediato; por su parte, en las clases medias y altas, los niños tendrían códigos más elaborados que expresan ideas abstractas con mayor facilidad que no está ligado a contextos particulares. De esta manera, dado que el proceso de aprendizaje maneja un código elaborado, un código restringido va a chocar con la cultura académica de la escuela, dándoles ventaja a los niños con códigos lingüísticos más elaborados que se adaptan fácilmente al entorno escolar.

Bourdieu y Passeron (1973), en las teorías de la reproducción cultural, explican cómo las escuelas y otras instituciones sociales perpetúan las desigualdades sociales y económicas. En este enfoque se recogen ideas de Bernstein (1975) e Illich (1974), quienes ven la escuela como un lugar de reproducción de valores dominantes y generadora de desigualdades sociales basadas en diferencias culturales como las formas de utilización del lenguaje.

La economía ha tratado de validar algunos de los postulados antes descritos; la economía laboral estudia la relación positiva entre los retornos al ingreso y los años de educación alcanzados; algunos resultados dicen que los retornos a la educación varían a través de la población al controlar factores como la calidad de la escuela y la educación parental (Card 1999). Las habilidades no cognitivas influencian fuertemente las decisiones de escolaridad, pero también afectan el salario futuro dadas las decisiones de educación (Heckman, Stixrud y Urzua 2006). Así mismo, Cunha y Heckman (2007) encuentran que las inversiones tempranas en formación de habilidades tienen un rendimiento mayor que las inversiones tardías; esto se debe a la autoproductividad y a la complementariedad dinámica de las inversiones -en un caso extremo este planteamiento lleva a concluir que remediar bajas inversiones tempranas es excesivamente costoso, si no imposible-. Así mismo, el entorno familiar afecta la formación de las habilidades ya que determina la inversión que podrían realizar los padres en sus hijos, además de acentuar el efecto de las restricciones de liquidez.

La creciente desigualdad en el ingreso en Estados Unidos durante los últimos cincuenta años ha traído consigo fuertes consecuencias en el desempeño 
académico. El estudio de Reardon (2012) resalta que las familias con mayores ingresos no solo tienen más dinero para invertir en el desarrollo cognitivo de sus hijos, sino que tienen acceso a otros recursos, familiares y sociales; por ejemplo, en las familias con mayor nivel socioeconómico, en promedio, los padres tienen mayores niveles de educación, lo que permite potenciar los desarrollos cognitivos de los hijos, situación que se refleja en los logros académicos. No obstante, los sociólogos han sostenido que los padres, en particular los de clase media, se han enfocado más en el desarrollo cognitivo de sus hijos en los últimos años. Reardon (2012) destaca el creciente aumento de la segregación residencial de los últimos años, las familias de elevados ingresos progresivamente viven espacialmente más alejadas de aquellas con ingresos intermedios y bajos.

La economía regional y urbana, de la mano de la teoría de la interacción social, evalúa las formas en que los factores personales tanto cognitivos como no cognitivos interactúan con el contexto de la clase durante el proceso de aprendizaje, e introducen el concepto de efecto compañeros de clase o "efecto pares" con el ánimo de evaluar el impacto de las interacciones entre los estudiantes sobre rendimiento académico. Arnott y Rowse (1987) analizan la asignación óptima de estudiantes y los gastos educativos dentro del aula de clase cuando los efectos pares están presentes; y concluyen que la función de producción educativa varía a medida que cambia la composición del grupo. Utilizando modelos multinivel, Garner y Raudenbush (1991) contrastan la hipótesis "habitar en un vecindario con carencias sociales tiene un efecto negativo sobre el desempeño académico de los estudiantes", y encuentran una amplia variación en el logro educativo entre vecindarios, la cual es explicada en una amplia variación, cuando es controlada por el entorno familiar.

Gaviria y Steven (2001) sostienen que los estudiantes están menos expuestos al entorno familiar de los pares de la escuela que lo que están al entorno familiar de sus pares que residen en el mismo vecindario. Basados en esto, argumentan que el efecto de las interacciones sociales observables en la escuela tiene mayor probabilidad de conducirlos por influencias bidireccionales (en lugar de efectos contextuales) que a ser efectos de interacción social como influencia del vecindario.

En Colombia, en un estudio que utiliza técnicas de modelos estructurales, Vivas, Correa y Domínguez (2011) corroboran que las realizaciones educativas potenciales generadas dentro de los hogares están estrechamente relacionadas con los efectos propios del entorno familiar y con la calidad de las vecindades; así mismo, en la medida que los entornos locales (efectos de vecindad) son más eficientes, mayores son los efectos sobre el potencial de logro educativo. Adicionalmente, el modelo indica que los individuos localizados en ambientes desfavorables, con jefatura del hogar femenina y entornos microlocales en los que prevalecen minorías étnicas con medios económicos escasos y entornos familiares y de vecindad precarios en los niveles educativos, cuentan con desventajas acumuladas que establecen una brecha importante frente a otros sujetos que se forman en ambientes propicios, ubicados en entornos socioeconómicos de elevada educación promedio de los familiares y vecinos (altas densidades de capital humano). 
En un estudio previo, Vivas (2008) encontró que los principales predictores de las diferencias observadas en los resultados obtenidos por los estudiantes en las pruebas de competencias educativas son -en cuanto al estudiante- las variables de contexto como el entorno familiar, los atributos socioeconómicos y sociodemográficos de los compañeros de clase, así como su entorno social, y -en cuanto al colegio- el clima educativo del plantel.

Otra línea que ha tenido alta influencia en el estudio de los factores que inciden en el desempeño académico es la psicología de la educación, al definir como punto de partida del razonamiento matemático y científico las capacidades cognitivas de los estudiantes. No obstante, hay ciertas variables internas de los estudiantes - cognitivas y no cognitivas (emocionales) - que inciden directamente en el proceso de aprendizaje, como las creencias, las representaciones y las valoraciones que tiene de sí mismo, las cuales son relevantes en el procesamiento de la información y en la regulación de la conducta, noción definida por Bandura (1993) como se mencionó anteriormente, autoeficacia percibida en su teoría del aprendizaje social.

La creencia en la autoeficacia en matemáticas tiene un impacto sobre el aprendizaje y el desempeño cognitivo, motivacional, emocional y afectivo. En particular, en el aspecto motivacional, determina el conjunto de metas fijadas por el estudiante, su grado de esfuerzo al resolver las tareas, la cantidad de tiempo que persevera en las fases de dificultad y el grado de resistencia al fracaso. El término autoeficacia se usa para describir la creencia de los estudiantes que, a través de sus acciones, pueden producir los efectos deseados, lo cual, a su vez, es un incentivo poderoso para actuar o perseverar haciendo frente a las dificultades (Bandura 1977). Altos niveles de autoeficacia pueden afectar la motivación en ambas vías, positiva y negativa. En general, las personas con alta autoeficacia tienen mayor probabilidad de hacer esfuerzos para completar una tarea y continuar más tiempo en esos esfuerzos, que aquellas con bajo nivel.

Para Bandura (1993) hay tres tipos de creencias en los que la autoeficacia percibida opera como un importante contribuyente al desarrollo académico: 1) las creencias del estudiante en su eficacia para regular su propio aprendizaje y dominar las actividades académicas, lo cual determina sus aspiraciones, su nivel de motivación y los logros académicos; 2) las creencias de los docentes en su eficacia personal para motivar y promover el aprendizaje, lo cual afecta a los tipos de ambientes de aprendizaje que se crean y el nivel de progreso académico de los estudiantes; 3) las creencias de las instituciones en su eficacia colectiva para la instrucción, lo que contribuye significativamente en su nivel de rendimiento académico.

La parte biológica de las capacidades cognitivas es estudiada por Spelke (2005); en su trabajo, la autora afirma que las capacidades cognitivas de base biológica son compartidas de forma equivalente por hombres y mujeres, que tanto los hombres como las mujeres presentan igual aptitud hacia las matemáticas y las ciencias, pero que esto no implica que la dotación genética de los seres humanos sea irrelevante para alcanzar mejores desempeños en estas áreas.

Una evaluación del desempeño académico según algunas características no cognitivas presentada por Niederle y Vesterlund (2010) permite concluir que en ambientes competitivos, como son las pruebas de desempeño tipo TIMSS, la 
brecha en matemáticas está a favor de los hombres, pero este resultado no se mantiene cuando la evaluación se da en ambientes no competitivos. La justificación es que adicional a las habilidades cognitivas, factores no cognitivos como la motivación, el comportamiento, la ansiedad, entre otros, puede afectar de manera importante el desempeño en la prueba.

Un estudio realizado por el ICFES (2013) presenta un exhaustivo análisis del comportamiento en el tiempo de la magnitud de las diferencias de género en Colombia, en las áreas de matemáticas y lenguaje, con base en diferentes fuentes de información nacional e internacional. La amplia revisión bibliográfica se complementa, en primer lugar, con la evaluación de las diferencias tipificadas de género, las cuales buscan evaluar la variación en la magnitud de la brecha de Colombia respecto a los países participantes en las pruebas internacionales TIMSS y Pisa, y entre las ciudades colombianas (pruebas Saber $5^{\circ}, 9^{\circ}$ y $11^{\circ}$ ), condicionadas por diferentes características como el tipo de colegio y el área de ubicación. Segundo, realiza la estimación de la brecha utilizando tanto análisis descriptivo como técnicas multinivel. El estudio concluye que las características de la escuela explican una alta proporción de las diferencias en los desempeños, que en el alumno los factores biopsicosociales son los que más influyen en las diferencias, y que en cuanto al colegio, el clima escolar es muy importante.

\section{Modelo analítico}

Los postulados de la teoría de la interacción social, formulados por Manski (1993), permiten construir el modelo analítico del estudio del desempeño académico. Tomando la propuesta desarrollada por Vivas (2008), se definen las componentes del modelo como: $R$ el vector de resultados en la prueba del estudiante $i$ que es explicado por el vector $F$ de variables socioeconómicas, por el vector $G$ de características del grupo de referencia, y por el vector $U$, que define la habilidad no observable del estudiante. El modelo en forma lineal se escribe como:

$$
\begin{aligned}
& R=a+\beta E[R \mid G]+E[F \mid G]^{\prime} \gamma+F^{\prime} \lambda+U, \\
& \text { donde } E[U \mid G, F]=G^{\prime} \delta
\end{aligned}
$$

Donde $E$ es la esperanza matemática. Al regresar en (1) $R$ sobre $(G, F)$ se obtiene la ecuación fundamental de interacción social:

$$
E(R \mid G, F)=\alpha+\beta E[R \mid G]+E[F \mid G]^{\prime} \gamma+F^{\prime} \lambda+G^{\prime} \delta
$$

Dados los vectores observados individuales y familiares $(R, G, F)$ y la variable $U$ no observada, se define el valor de proficiencia esperado, $\operatorname{con}(\alpha, \beta, \gamma, \lambda, \delta)$ el vector de parámetros del modelo. La especificación (2) intenta capturar los efectos endógenos $(\beta)$, contextuales $(\gamma)$ y correlacionados $(\delta)$, así como los efectos directos del entorno familiar $(\lambda)$.

Son varios los problemas que se pueden presentar; en primer lugar, si los efectos correlacionados son significativos, $(\delta \neq 0)$, implica que los individuos que 
conforman el grupo tienden a comportarse o exhibir resultados similares en el atributo no observado $U$. Segundo, dado que tanto $E[R \mid G]$ como $E[F \mid G]$ dependen de $G$ está latente en el modelo el problema de identificación en el sentido que le confiere Manski (1993), lo que puede generar un problema de colinealidad perfecta y por tanto que los parámetros no puedan ser identificados y así los efectos endógenos puros no puedan distinguirse de los contextuales ni de los correlacionados.

Luego de eliminar el problema de colinealidad perfecta, los parámetros quedan identificados, la dificultad es que no es posible distinguir entre los efectos endógenos puros y los contextuales, pero es posible capturar la incidencia de los factores sociales o del grupo de referencia.

Otra solución es suponer que $\delta=\gamma=0$ (efectos contextuales y correlacionados nulos) y obtener, luego de eliminar el problema de colinealidad, un modelo completamente endógeno con parámetros exactamente identificados. Bajo este supuesto se tendría una aproximación indirecta de la magnitud de los efectos endógenos $(\beta)$ y una directa del entorno familiar $(\lambda)$ (Vivas 2008).

Una especificación compacta para la realización de los ejercicios econométricos que expresa la forma reducida sería:

$$
R_{i j}=\varphi+\lambda F_{i j}+\phi S h c_{j}+\xi \bar{F}_{(-i) j}+\mu_{j}+\varepsilon_{i j}
$$

Donde $R_{i j}$ define los puntajes en la prueba del estudiante i que pertenece a la escuela $j ; F_{i j}$ con̄tiene las características individuales y las variables de nivel socioeconómico; $F_{(-i) j}$ representa el promedio del nivel socioeconómico de los compañeros de clase (excluyendo al individuo $i$ ); $S h c_{j}$ define el conjunto de variables asociados a la escuela $j ; \mu_{j}$ y $\varepsilon_{i j}$ son los errores aleatorios en el aula, la escuela y los individuales, respectivamente. $\lambda, \phi$ y $\xi$ son los parámetros asociados al entorno familiar, al aula o escuela y a los atributos de los compañeros de clase, respectivamente.

\section{Materiales y métodos}

\subsection{Datos y variables}

El estudio TIMSS (Trends in International Mathematics and Science Study) es una evaluación internacional que mide las tendencias de los desempeños en matemáticas y ciencias al finalizar los grados cuarto y octavo en un amplio número de países en el mundo, cuyo objetivo es proveer datos comparativos acerca de los logros educativos en diferentes países con el fin de mejorar la enseñanza y el aprendizaje de las matemáticas y las ciencias. TIMSS monitorea la cobertura del currículo y su implementación. En las pruebas realizadas por la IEA (International Association for the Evaluation of Educational Achievement), Colombia solo ha participado en los años 1995 y 2007. En el 2007 participaron 59 países con cerca de 425.000 estudiantes. En Colombia, la prueba fue aplicada a 4.831 alumnos de cuarto grado en 142 colegios y a 4.873 de octavo en 148 .

El proyecto TIMSS 2007 se basa en el currículo, entendido como el principal concepto organizador para establecer qué saben los estudiantes y cuáles son los 
factores que inciden en sus aprendizajes. La prueba evalúa dominios cognitivos que corresponden a las destrezas y habilidades asociadas a los conocimientos concretos del estudiante, clasificados en tres componentes: conocer, razonar y aplicar.

Para el análisis de los resultados se tendrá en cuenta variables que tienen que ver tanto con el estudiante como con el colegio, con el fin de explicar la variación en los resultados de ambos. A continuación se realiza una descripción de las variables a utilizar para explicar las diferencias en el desempeño en la prueba.

- Desempeño en matemáticas: es la variable a explicar, mide el nivel de proficiencia de los estudiantes de cuarto y octavo grados en la prueba de matemáticas.

- Sexo: variable dicotómica que relaciona el sexo del estudiante, creada con el valor uno para los hombres y cero para las niñas.

- Sector: variable dicotómica que define el carácter público o privado del colegio, toma el valor uno si el colegio es privado y cero si es público.

- Índice socioeconómico (ISE): índice estandarizado, con media cero y desviación estándar uno. Construido a partir de las variables: recursos del hogar como la posesión de libros, computadores y/o salas de estudio, nivel educativo de los padres (octavo grado).

- Índice de los compañeros de clase (efecto par): es un indicador del desempeño medio de los pares escolares del estudiante i. Construida a partir del promedio por colegio del ISE de los estudiantes menos el efecto del estudiante $i$.

- Índice de autoconfianza de los estudiantes del aprendizaje de las matemáticas (ACM): creado por TIMSS para investigar cómo piensan los estudiantes acerca de sus habilidades en matemáticas. Basado en las respuestas de cuatro preguntas: $i$.) Usualmente lo hago bien en matemáticas. ii.) Las matemáticas son más duras para mí que para muchos de mis compañeros de clase. iii.) No soy bueno en matemáticas. iv.) Aprendo cosas rápidamente en matemáticas.

Adicionalmente se incluyen variables de interacción entre el género, el sector y el índice de autoconfianza con el fin de evaluar los efectos diferenciales entre estos grupos. Además de los signos positivos asociados a los hombres y a los colegios privados, se espera que a medida que se incrementa en una unidad el valor de cada uno de los índices el desempeño académico de los estudiantes también se incremente.

\subsection{Metodología}

La estructura jerárquica que presentan los datos de las pruebas TIMMS permite utilizar una estrategia de análisis en ambos niveles de agregación, buscando eliminar potenciales problemas tanto de estimación como de inferencia.

El modelo multinivel corrige los problemas de agregación así como de no independencia de las observaciones (Longford y Aikkin 1986). Además, permite estudiar por separado la varianza de los desempeños dentro de cada colegio y entre los colegios, siendo así la metodología de análisis más adecuada para tratar datos jerarquizados.

Para estudiar la relación entre los estudiantes y el colegio, se utiliza un modelo de regresión lineal jerárquico de dos niveles. La prueba TIMSS define una estructura jerárquica con ordenamiento perfecto de tres niveles de agregación, 
estudiantes anidados en aulas de clase, y estas anidadas en colegios. En este estudio se utilizan dos niveles de agregación, los estudiantes que se agrupan en los colegios. Asumiendo que $i$ representa las unidades del nivel inferior o de estudiantes y $j$ las del nivel superior o de colegio y suponiendo, sin pérdida de generalidad, dos variables regresoras, se define el modelo:

$$
y_{i j}=\beta_{0 j}+\beta_{1 j} x_{1 i j}+\beta_{2 j} x_{2 i j}+\varepsilon_{i j}
$$

Donde, $y_{i j}$ es el logro alcanzado por el estudiante $i$ en la escuela $j$. ( $i=1, \ldots, n$; $j=1, \ldots, j)$. El vector $x_{i j}=\left\{x_{1 i j}, x_{2 i}, \ldots, x_{k i j}\right\}$ representa las variables de contexto, en cuanto al estudiante, el profesor y la institución que explican las variaciones en el desempeño. $\varepsilon_{i j}$ es el error estocástico normal e independientemente distribuido con media cero y varianza $\sigma_{\varepsilon \circ}^{2}$ constante. Suponiendo que los parámetros $\beta_{k j}$ son variables aleatorias, se tiene

$$
\beta_{0 j}=\beta_{0}+\mu_{0 j} \quad \beta_{1 j}=\beta_{1}+\mu_{1 j} \quad \beta_{2 j}=\beta_{2}+\mu_{2 j}
$$

Donde $\mu_{o j} \mu_{1 j} y \mu_{2 j}$ son variables aleatorias que se distribuyen conjuntamente normal con matriz de varianza covarianza, $\Omega_{\mu}$ definida como:

$$
\left[\begin{array}{l}
\mu_{0 j} \\
\mu_{1 j} \\
\mu_{2 j}
\end{array}\right] \sim N\left(0, \sigma^{2} \Omega_{\mu}\right) \quad \text { con } \Omega_{\mu}=\left[\begin{array}{ccc}
\sigma_{\mu_{0}}^{2} & \sigma_{\mu_{01}}^{2} & \sigma_{\mu_{02}}^{2} \\
\sigma_{\mu_{10}}^{2} & \sigma_{\mu_{1}}^{2} & \sigma_{\mu_{12}}^{2} \\
\sigma_{\mu_{20}}^{2} & \sigma_{\mu_{21}}^{2} & \sigma_{\mu_{2}}^{2}
\end{array}\right]
$$

Reemplazando (2) en (1) se obtiene:

$$
y_{i j}=\beta_{0}+\beta_{1} x_{1 i j}+\beta_{2} x_{2 i j}+\left(\mu_{0 j}+\mu_{1 j} x_{1 i j}+\mu_{2 j} x_{2 i j}+\varepsilon_{0 i j}\right)
$$

El modelo (4) requiere de la estimación de tres parámetros fijos $\beta_{0}, \beta_{1}$ y $\beta_{2} \mathrm{y}$ los seis parámetros de la matriz de covarianzas $\Omega_{\mu}$. Suponiendo que no se tienen variables regresoras, el modelo (4) toma la forma:

$$
y_{i j}=\beta_{0}+\mu_{0 j}+\varepsilon_{0 i j}
$$

Llamado modelo nulo o modelo de componentes de varianza, ya que

$\operatorname{Var}\left(y_{i j} \mid \beta_{k}, x_{i j}\right)=\sigma_{\mu_{0}}^{2}+\sigma_{\varepsilon_{0}}^{2}$

Y la covarianza entre dos estudiantes $i_{1}$ y $i_{2}$ del mismo colegio, está dada por:

$\operatorname{Cov}\left(\mu_{0 j}+\varepsilon_{0 i_{1} j}, \mu_{0 j}+\varepsilon_{0 i_{2} j}\right)=\sigma_{\mu_{0}}^{2}$

Dado que se asume que los residuales del nivel 1 son independientes, la correlación entre estos dos estudiantes se define como:

$$
\rho=\frac{\sigma_{\mu_{0}}^{2}}{\sigma_{\mu_{o}}^{2}+\sigma_{\varepsilon_{0}}^{2}}
$$

llamado coeficiente de correlación intraclase (ICC), en este caso intracolegios; mide la proporción de la varianza total que es debida a los colegios. La existencia de un ICC diferente de cero significa que el método de estimación de mínimos cuadrados no es adecuado ya que conduce a inferencias incorrectas. 


\section{Análisis de resultados}

Existe una amplia relación entre el desempeño en lectura y el de matemáticas en especial en los niveles de más baja formación; un estudiante que tiene problemas en lectura difícilmente podrá tener una óptima comprensión de los enunciados en matemáticas. Este razonamiento nos lleva a proponer un análisis general del desempeño de los estudiantes en lectura antes de realizar el respectivo estudio de los resultados en matemáticas. Los datos utilizados provienen de las prueba TIMSS 2007 desarrollada por la IEA.

\subsection{Análisis descriptivo}

En la prueba TIMSS 2007, tanto en grado cuarto como en octavo el desempeño de los estudiantes colombianos en el área de matemáticas fue bastante bajo al ser comparado con el promedio de los países de la OCDE (500 puntos) y con el promedio de los países asiáticos, que obtuvieron los mejores resultados. En cuarto grado, Colombia obtuvo un desempeño promedio de 355,7 puntos con una desviación estándar de 84,7 puntos, puntaje ampliamente superado por Hong Kong (607), Singapur (599), Taipéi (576) y Japón (568); y superó solamente a Marruecos (341), El Salvador (330), Túnez (327), Kuwait (316), Qatar (296) y Yemen (224). En octavo, la situación mejora levemente, pero el logro promedio de 379,6 puntos con desviación estándar de 74,2 puntos es muy bajo al compararlo con el obtenido en Taipéi (598), Corea (597) y Singapur (593) y los países de la OCDE (500).

Por género, los hombres alcanzaron desempeños más altos en matemáticas que las mujeres; la tabla 1 deja ver que en cuarto grado la diferencia fue de 17 puntos, mientras que en octavo la brecha alcanzó 31,8 puntos en promedio. Colombia, entre los países participantes en la prueba, registra el más alto diferencial a favor de los hombres, no obstante que en más de la mitad de los países la diferencia fue no significativa y en ocho de ellos la brecha estuvo a favor de las mujeres (Mullis, Martin y Foy 2008).

Tabla 1. Desempeño en matemáticas Prueba TIMSS 2007

\begin{tabular}{|c|l|r|r|r|r|r|r|}
\hline Prueba & \multicolumn{1}{|c|}{ Se:co } & Promedio & $\begin{array}{c}\text { Desviación } \\
\text { estándar }\end{array}$ & $\begin{array}{c}\text { Valor } \\
\text { minimo }\end{array}$ & $\begin{array}{c}\text { Valor } \\
\text { máximo }\end{array}$ & Muestra & Proporción \\
\hline \multirow{4}{*}{$\begin{array}{c}\text { Cuarto } \\
\text { grado }\end{array}$} & Total & $\mathbf{3 5 5 , 7}$ & 84,62 & 91,1 & 714,8 & 4.831 & $100,0 \%$ \\
\cline { 2 - 8 } & Hombre & $\mathbf{3 6 4 , 4}$ & 87,75 & 127,5 & 714,8 & 2.415 & $50,0 \%$ \\
\cline { 2 - 8 } & Mujer & $\mathbf{3 4 7 , 0}$ & 80,40 & 91,1 & 620,7 & 2.416 & $50,0 \%$ \\
\cline { 2 - 8 } & Diferencia & $\mathbf{1 7 , 4}$ & & 36,4 & 94,1 & & \\
\hline \multirow{3}{*}{$\begin{array}{c}\text { Octavo } \\
\text { grado }\end{array}$} & Total & $\mathbf{3 7 9 , 6}$ & 74,19 & 125,3 & 726,2 & 4.873 & $100,0 \%$ \\
\cline { 2 - 8 } & Hombre & $\mathbf{3 9 5 , 8}$ & 76,12 & 138,0 & 726,2 & 2.389 & $49,0 \%$ \\
\cline { 2 - 8 } & Mujer & $\mathbf{3 6 4 , 1}$ & 68,80 & 125,3 & 568,8 & 2.484 & $51,0 \%$ \\
\cline { 2 - 8 } & Diferencia & $\mathbf{3 1 , 8}$ & & 12,7 & 157,4 & & \\
\hline
\end{tabular}

Fuente: Prueba TIMSS 2007. Cálculos del autor.

La gráfica 1 describe la distribución del desempeño de los estudiantes de los grados cuarto y octavo; es clara la hegemonía de los chicos en todos los puntos de la 
distribución, no obstante los de cuarto grado presentan una brecha más ajustada en el $50 \%$ inferior de los puntajes, de ahí en adelante, a medida que mejoran los puntajes las diferencias se van haciendo más amplias. En octavo grado la tendencia es más clara, la brecha en los puntajes entre chicos y chicas está claramente definida desde los puntos más bajos aumentando según incrementa el desempeño de los estudiantes.

Gráfica 1. Distribución del desempeño en Matemáticas Prueba TIMSS 2007
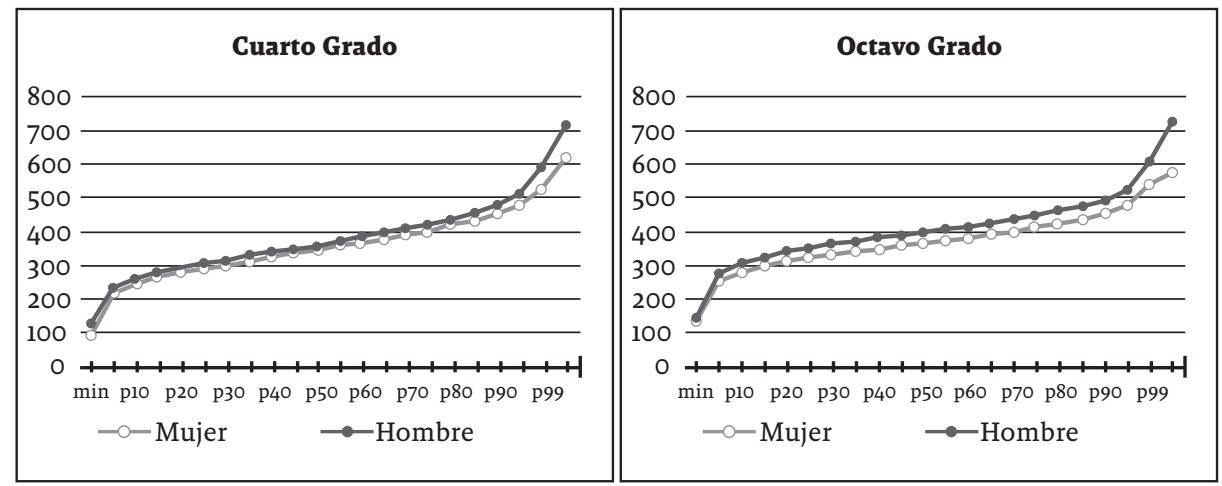

Fuente: Prueba TIMSS 2007. Cálculos del autor.

La prueba TIMSS identifica cuatro puntos en la escala global de matemáticas que sirven de comparación en los estándares internacionales; los valores 625, 550, 475, y 400 definen respectivamente los intervalos correspondientes a los niveles de desempeño: avanzado, alto, intermedio y bajo. Los puntajes por debajo de 400 definen otra categoría llamada inferior. La tabla 2 presenta la definición general que establece en cada nivel, lo que saben y deben hacer los estudiantes en un contexto determinado.

Tabla 2. Niveles de desempeño en la prueba de matemáticas.

¿Qué saben y deben hacer los estudiantes en cada nivel?

\begin{tabular}{|c|c|c|}
\hline $\begin{array}{l}\text { Nivel de } \\
\text { desempeño }\end{array}$ & Cuarto grado & Octavo grado \\
\hline $\begin{array}{c}\text { Avanzado } \\
\text { (625 puntos o más) }\end{array}$ & $\begin{array}{l}\text { Aplican su conocimiento y } \\
\text { comprensión en una variedad de } \\
\text { situaciones relativamente complejas y } \\
\text { explican su razonamiento }\end{array}$ & $\begin{array}{l}\text { Organizan y plantean } \\
\text { conclusiones a partir de } \\
\text { información, hacen } \\
\text { generalizaciones y resuelven } \\
\text { problemas no rutinarios }\end{array}$ \\
\hline $\begin{array}{c}\text { Alto } \\
\text { (550-624 puntos) }\end{array}$ & $\begin{array}{l}\text { Aplican su conocimiento y } \\
\text { comprensión para resolver problemas }\end{array}$ & $\begin{array}{l}\text { Aplican su conocimiento y } \\
\text { comprensión en situaciones } \\
\text { relativamente complejas }\end{array}$ \\
\hline $\begin{array}{c}\text { Medio } \\
(475-549 \text { puntos })\end{array}$ & $\begin{array}{l}\text { Aplican conocimiento matemático } \\
\text { básico en situaciones simples }\end{array}$ & $\begin{array}{l}\text { Aplican conocimiento matemático } \\
\text { básico en situaciones simples }\end{array}$ \\
\hline $\begin{array}{c}\text { Bajo } \\
(400-474 \text { puntos })\end{array}$ & $\begin{array}{l}\text { Tienen un conocimiento básico de las } \\
\text { matemáticas }\end{array}$ & $\begin{array}{l}\text { Tienen algún conocimiento sobre } \\
\text { números naturales y decimales, } \\
\text { operaciones y gráficos }\end{array}$ \\
\hline $\begin{array}{c}\text { Inferior } \\
\text { (399 puntos o menos) }\end{array}$ & $\begin{array}{l}\text { Tienen un conocimiento matemático } \\
\text { inferior al mínimo que permite } \\
\text { describir la prueba TIMSS } 2007\end{array}$ & $\begin{array}{l}\text { Tienen un conocimiento } \\
\text { matemático inferior al mínimo } \\
\text { que permite describir la prueba } \\
\text { TIMSS } 2007\end{array}$ \\
\hline
\end{tabular}

Fuente: Mullis, Martin y Foy (2008, 68-69). 
La tabla 3 muestra el desempeño medio en la prueba de matemáticas de los estudiantes colombianos de cuarto y octavo grados. La parte negativa de los resultados es que más de $60 \%$ de los estudiantes evaluados de ambos grados presentan un nivel de conocimiento matemático inferior al mínimo descrito en la prueba TIMSS, y que $\mathbf{2 5 , 6 3 \%}$ en cuarto grado y $\mathbf{2 9} \%$ en octavo apenas alcanzan un conocimiento básico. En los niveles medio, alto y avanzado, tanto en cuarto como en octavo, solo se ubica un poco más de $10 \%$ de los estudiantes, donde $8,8 \%$ aplica solo conocimientos matemáticos básicos en situaciones simples, $1,5 \%$ lo aplica en situaciones relativamente complejas y el restante $0,33 \%$ (en cuarto) y 0,35\% (en octavo) los utilizan en situaciones complejas.

Tabla 3. Resultados por niveles de desempeño para los grados cuarto y octavo, prueba TIMSS 2007

\begin{tabular}{|c|c|c|c|c|c|c|c|}
\hline \multirow{2}{*}{$\begin{array}{l}\text { Puntaje } \\
\text { limite } \\
\text { inferior }\end{array}$} & \multirow[b]{2}{*}{ Niveles } & \multicolumn{3}{|c|}{ Cuarto grado } & \multicolumn{3}{|c|}{ Octavo grado } \\
\hline & & $\begin{array}{l}\text { Número de } \\
\text { estudiante }\end{array}$ & $\begin{array}{c}\text { Porcentaje } \\
\text { por nivel }\end{array}$ & $\begin{array}{l}\text { Porcentaje } \\
\text { acumulado }\end{array}$ & $\begin{array}{l}\text { Número de } \\
\text { estudiante }\end{array}$ & $\begin{array}{c}\text { Porcentaje } \\
\text { por nivel }\end{array}$ & $\begin{array}{l}\text { Porcentaje } \\
\text { acumulado }\end{array}$ \\
\hline Menos de 400 & Inferior & 3.075 & 63,65 & 63,65 & 2.945 & 60,43 & 60,43 \\
\hline Menos de 475 & Bajo & 1.238 & 25,63 & 89,28 & 1.415 & 29,03 & 89,46 \\
\hline Menos de 550 & Medio & 427 & 8,84 & 98,12 & 426 & 8,74 & 98,20 \\
\hline Menos de 625 & Alto & 75 & 1,55 & 99,67 & 70 & 1,45 & 99,65 \\
\hline 625 o más & Avanzado & 16 & 0,33 & 100,00 & 17 & 0,35 & 100,00 \\
\hline
\end{tabular}

Fuente: Prueba TIMSS 2007. Cálculos del autor.

La evaluación del desempeño en la prueba de matemáticas luego de controlar por las variables contextuales de género y el tipo de institución (pública o privada) se presenta en la tabla 4. Por género, tanto en los colegios oficiales como en los privados, los niños obtuvieron un desempeño más alto en la prueba que las niñas; se observa además que la dispersión de los promedios disminuye en el grado octavo respecto al cuarto, es decir, los resultados se encuentran más concentrados alrededor de la media, pero la variabilidad de los puntajes de los hombres, en particular en los colegios privados, es mayor. Es decir, los chicos de los colegios privados alcanzan los logros más sobresalientes en la prueba. Este resultado es similar al planteado por Ellison y Swanson (2010), quienes afirman que en la cola más alta de la distribución, el desempeño en matemáticas de los chicos es más sobresaliente que el de las chicas.

$\mathrm{Al}$ evaluar las brechas entre los promedios de desempeño entre niños y niñas, se observa que en los colegios oficiales es de 13,3 puntos en cuarto grado, y que en octavo asciende a 29 puntos, en promedio. En los colegios privados, la brecha tiende a ser constante, pero ya en cuarto grado se evidencia un amplio diferencial a favor de los niños (30,5 y 35,1 puntos en cuarto y octavo respectivamente); este resultado hace pensar que en los colegios privados la construcción de autoconceptos en matemáticas por parte de los estudiantes se presenta a más temprana edad, o que en promedio la institución le permite al estudiante identificar a más temprana edad las áreas de mayor fortaleza. 
Tabla 4. Desempeño promedio en matemáticas según sector y sexo TIMSS 2007

\begin{tabular}{|c|c|c|c|c|c|c|c|}
\hline \multirow{2}{*}{ Género } & \multirow{2}{*}{ Estadísticos } & \multicolumn{3}{|c|}{ Cuarto grado } & \multicolumn{3}{|c|}{ Octavo grado } \\
\hline & & Oficial & Privado & Total & Oficial & Privado & Total \\
\hline \multirow{4}{*}{ Mujer } & Promedio & 338,4 & 393,6 & 347,0 & 354,7 & 409,4 & 364,1 \\
\hline & Error estándar & 78,0 & 77,2 & 80,4 & 65,2 & 68,0 & 68,8 \\
\hline & Observaciones & 2.038 & 378 & 2.416 & 2.089 & 395 & 2.484 \\
\hline & Proporción & $84,4 \%$ & $15,6 \%$ & $50,0 \%$ & $84,1 \%$ & $15,9 \%$ & $51,0 \%$ \\
\hline \multirow{4}{*}{ Hombre } & Promedio & 351,7 & 424,0 & 364,4 & 384,6 & 444,4 & 395,8 \\
\hline & Error estándar & 80,9 & 93,9 & 87,7 & 69,6 & 83,8 & 76,1 \\
\hline & Observaciones & 1.992 & 423 & 2.415 & 1.940 & 449 & 2.389 \\
\hline & Proporción & $82,5 \%$ & $17,5 \%$ & $50,0 \%$ & $81,2 \%$ & $18,8 \%$ & $49,0 \%$ \\
\hline \multirow{4}{*}{ Total } & Promedio & 345,0 & 409,9 & 355,7 & 369,2 & 427,4 & 379,6 \\
\hline & Error estándar & 79,8 & 87,8 & 84,6 & 69,0 & 78,5 & 74,2 \\
\hline & Observaciones & 4.030 & 801 & 4.831 & 4.029 & 844 & 4.873 \\
\hline & Proporción & $83,4 \%$ & $16,6 \%$ & $100,0 \%$ & $82,7 \%$ & $17,3 \%$ & $100,0 \%$ \\
\hline
\end{tabular}

Fuente: Prueba TIMSS 2007. Cálculos del autor.

Por sector, los colegios privados alcanzaron en promedio 64,9 puntos más que los públicos. En los colegios privados, las niñas tanto en cuarto grado como en octavo alcanzaron cerca de 55 puntos de diferencia promedio a favor, respecto a sus homólogas de los colegios públicos. Los niños de las instituciones privadas de cuarto grado alcanzaron un diferencial a favor de 73 puntos, mientras que en octavo la brecha fue de 60 puntos, en promedio.

\subsection{Comportamiento de los índices}

La prueba TIMSS 2007 define un amplio número de índices discretos que permiten evaluar tanto el efecto directo sobre el desempeño en la prueba como el impacto diferencial de la interacción del índice con algunas de las variables del nivel estudiante o del colegio. Adicionalmente, la base proporciona la información necesaria para construir algún índice adicional, como es el caso con el índice socioeconómico, que fue construido con base en las variables de posesiones materiales del hogar, número de libros en el hogar y nivel de educación de los padres en el caso de los estudiantes de octavo.

\subsection{1 Índice de nivel socioeconómico (ISE)}

El nivel socioeconómico del hogar es una de las variables más utilizadas en los estudios de logros educativos, desde la teoría del capital humano podemos afirmar que existe una relación positiva entre el nivel de capital humano y el nivel socioeconómico del hogar. Existe además un amplio consenso en que la naturaleza del estatus socioeconómico está relacionada con la renta media 
familiar, el nivel educativo de los padres y su ocupación; padres con altos niveles de educación proveen mayores recursos y oportunidades a sus hijos para que desarrollen habilidades cognitivas y por ende mejores desempeños, que los padres menos educados (Reardon 2012).

Los diagramas de puntos de la gráfica 2 permiten evaluar la relación entre el desempeño académico y el nivel socioeconómico de los estudiantes controlado por el tipo de institución pública-privada. En los diagramas, la línea horizontal punteada representa el promedio de los países de la Organización para la Cooperación y el Desarrollo Económico (OECD por su sigla en inglés) (500 puntos) y la línea continua, el promedio para Colombia 355,7 puntos en cuarto grado y 379,6 puntos en octavo); la línea vertical representa la valor medio del nivel socioeconómico que en ambos casos se encuentra por debajo del valor cero. Además, los puntos de color negros en los diagramas representan los puntajes de los estudiantes pertenecientes a los colegios privados, mientras que los de color gris están asociados con los colegios públicos.

Gráfica 2. Desempeño en matemáticas versus índice socioeconómico del estudiante según sector

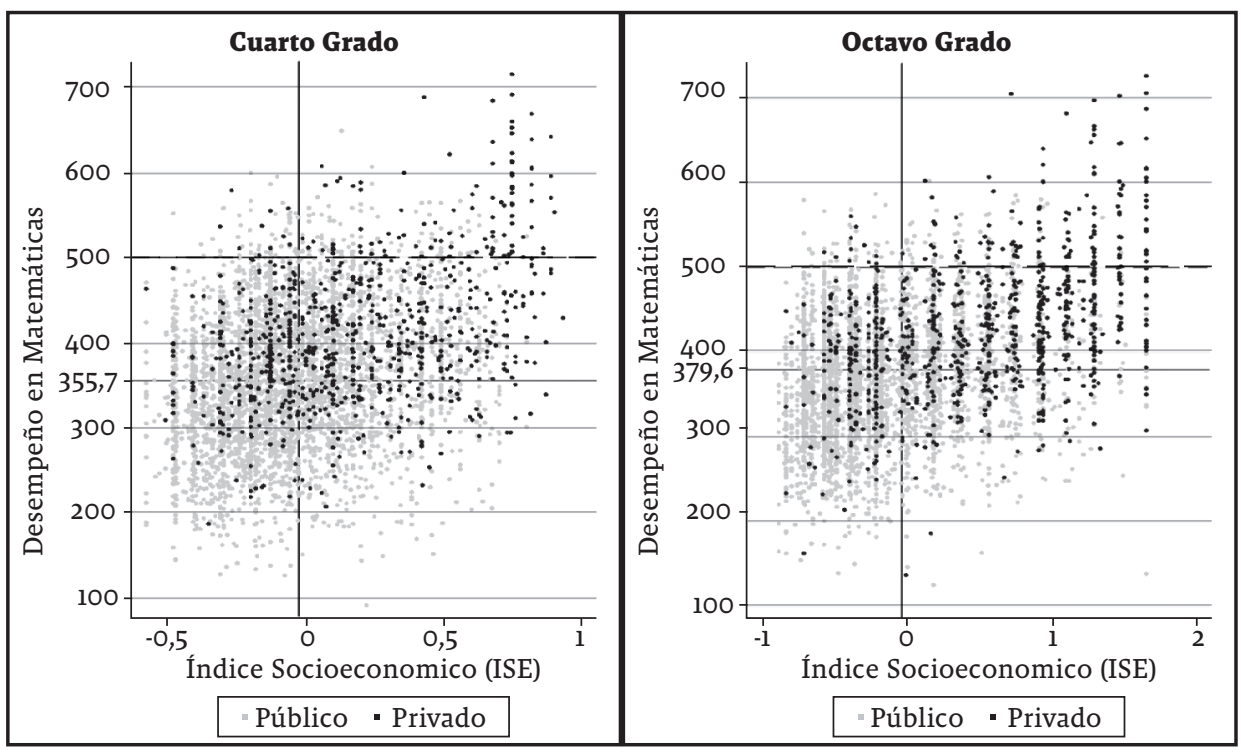

Fuente: Prueba TIMSS 2007. Cálculos del autor.

De la relación entre los puntajes en la prueba y el ISE lo primero a resaltar es la tendencia creciente de los puntajes respecto a incrementos en el ISE que se presenta tanto en cuarto grado como en octavo. Se corrobora la hipótesis de que los estudiantes de entornos socioeconómicos más aventajados en general alcanzan mejores desempeños académicos.

$\mathrm{Al}$ intersecarse las líneas: promedio de los países de la OECD —línea horizontal- con el promedio del ISE - línea vertical- se forma un plano de cuatro cuadrantes que permiten resaltar varios puntos. i.) Los estudiantes con mejores puntajes y más alto nivel socioeconómico se ubican en el primer cuadrante, 
formando una nube donde sobresalen los puntos negros que corresponden a los colegios privados; no obstante, en cuarto grado este cuadrante tiene una alta representación de puntos de color gris, indicando que en el rendimiento intervienen otras características además del nivel socioeconómico. ii.) La franja entre las dos líneas horizontales — promedio de Colombia y de la OECD— está representada mayoritariamente por estudiantes de colegios privados. iii.) Los cuadrantes por debajo de la línea que representa el promedio de Colombia concentran una alta proporción de puntos de color gris, es decir, estudiantes que tienen bajo nivel socioeconómico y bajo desempeño académico, lo que confirma que los bajos puntajes son más recurrentes en los colegios públicos.

Una estimación del valor de la pendiente de la relación entre el desempeño académico y el nivel socioeconómico indica que el ISE, en promedio, explica $26 \%$ de las variaciones en los puntajes, proporción relativamente baja; luego se requiere explorar otras variables, adicionales al ISE, que se espera expliquen la variación en los puntajes en las pruebas de matemáticas de cuarto y octavo, en cuanto al estudiante y al colegio.

\subsubsection{Efecto compañeros de clase (efecto de los pares)}

La familia es fundamental en la formación de las habilidades y capacidades de los hijos; además de las influencias genéticas, las inversiones tempranas por parte de los padres en la formación de habilidades, el diálogo constante entre hijos y padres, el entorno del hogar, entre otras, hacen parte del conjunto de características que Bourdieu denomina capital social "conjunto de recursos reales o potenciales a disposición de una red duradera de relaciones más o menos institucionalizadas de conocimiento o reconocimiento mutuo" (Bourdieu 2000, 148). Más adelante, este autor, de la mano de las teorías de la reproducción cultural, explica cómo las escuelas y otras instituciones sociales perpetúan las desigualdades sociales y económicas (Bourdieu y Passeron 1973).

El contexto social al que pertenece el estudiante determina el tipo de colegio al que este asiste, y las características demográficas del aula de clase o de la escuela ejercen un efecto sobre el desempeño académico superior al efecto del entorno familiar del alumno. Este resultado es utilizado por Manski (1993) para evaluar la propensión a que un estudiante se comporte de algún modo específico según el comportamiento predominante del grupo de referencia (efectos endógenos), pero el comportamiento del grupo de referencia puede estar influenciado por una característica exógena (efectos exógenos). Por último, estudiantes del mismo grupo de referencia tienden a comportarse de modo semejante bien sea porque afrontan ambientes similares y/o porque tienen características personales similares (efectos correlacionados).

$\mathrm{Al}$ evaluar la relación entre el desempeño en matemáticas y el efecto de los pares en la clase por tipo de colegio (gráfica 3) se observa una partición producto de la interacción de las variables. El efecto de los pares segmenta los puntos colocando en la parte baja de la distribución a los colegios públicos -nube de puntos gris con valores por debajo de 60 puntos en el eje horizontal, tanto en cuarto como en octavo-, mientras que en los colegios privados, los puntos se 
concentran en la parte alta de la distribución. Dado que la variable efecto de los pares es utilizada como una proxy del capital social de los estudiantes, la gráfica 3 muestra que a medida que el efecto de los pares o el capital social se incrementa, el desempeño académico de los estudiantes se incrementa a tasas bastante altas, producto de la alta inclinación de la recta.

Gráfica 3. Distribución del desempeño académico de los estudiantes versus efecto compañeros de clase según niveles de desempeño controlado por sector

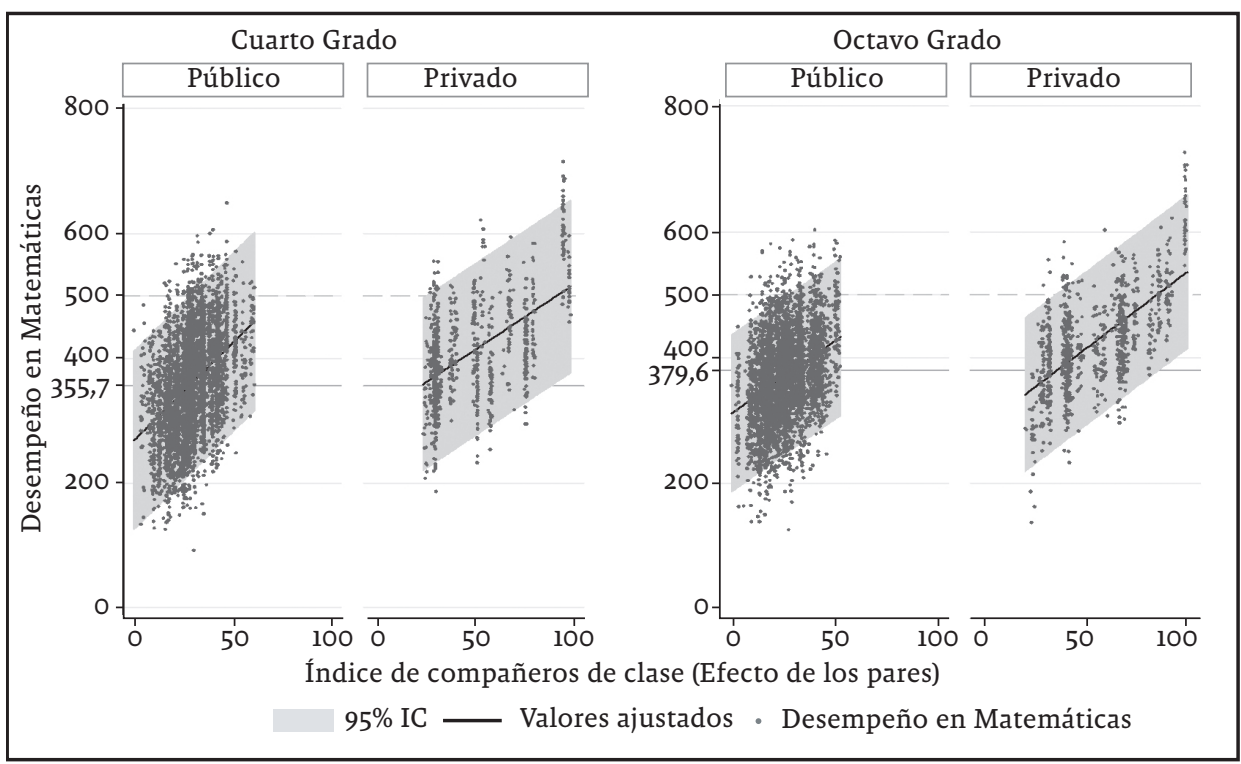

Fuente: Prueba TIMSS 2007. Cálculos del autor

La pregunta sería ¿el aumento en el desempeño se debe a efectos endógenos, exógenos o correlacionados? Como lo plantea Manski (1993), es imposible determinar tal comportamiento a partir únicamente de los datos. Es necesario tener en cuenta características adicionales, por ejemplo, el conjunto de recursos reales más que los potenciales con que llegan los estudiantes al colegio; en este contexto, dado que los colegios públicos albergan predominantemente estudiantes de bajo nivel socioeconómico cuyos padres tienen bajo nivel de educación, que viven en ambientes hostiles, el entorno del estudiante y el grupo de referencia en ambiente escolar influye negativamente en el rendimiento promedio de un estudiante particular. Por su parte, los estudiantes de los colegios privados se comportan de forma similar, dado que provienen de hogares con ambientes similares y tienen características personales similares. En resumen, se puede afirmar que en los colegios públicos influye una combinación de ambos efectos, endógenos y exógenos, mientras que en los privados los correlacionados tienen mayor predominio, no siendo un multiplicador social que necesariamente proporcione un impacto positivo sobre el desempeño de los estudiantes. 


\subsubsection{El índice de autoconfianza de los estudiantes en matemáticas}

La autoconfianza en matemáticas se define como el sentimiento de autocreencia de poder resolver los problemas o tareas en matemáticas. Por tanto, niveles bajos de autoconfianza están asociados con la falta de motivación, impotencia, ansiedad, frustración e inseguridad, lo que lleva al estudiante a experimentar bajo nivel de autoeficacia en matemáticas, impidiéndole afrontar con éxito las tareas.

La gráfica 4 describe la proporción de estudiantes clasificados por nivel: alto, medio y bajo en el índice de autoconfianza en matemáticas (ver anexo 1). Se observa que los chicos tienen mayor presencia en la categoría alto nivel de autoconfianza y menor en la de bajo nivel; además, dado que la formación de autoconceptos es un proceso dinámico, la tendencia en la proporción varía de cuarto a octavo, incrementando el número de estudiantes hombres en el nivel alto (pasa de $52,6 \%$ a 54\%) y disminuyendo en la categoría bajo (pasa de $44,5 \%$ a $36,5 \%$ ); en las chicas el comportamiento es a la inversa, es decir mientras que los chicos entre cuarto y octavo forman autoconceptos mejorando la autoconfianza en el desempeño en matemáticas, en las chicas la asignación de valores es negativa, por tanto terminan considerando que no son buenas para las matemáticas, que su proceso de aprendizaje es muy lento o que las matemáticas son muy difíciles, lo cual afecta su rendimiento.

Gráfica 4. Proporción de estudiantes en cada nivel del índice de autoconfianza en el aprendizaje de las matemáticas según sexo

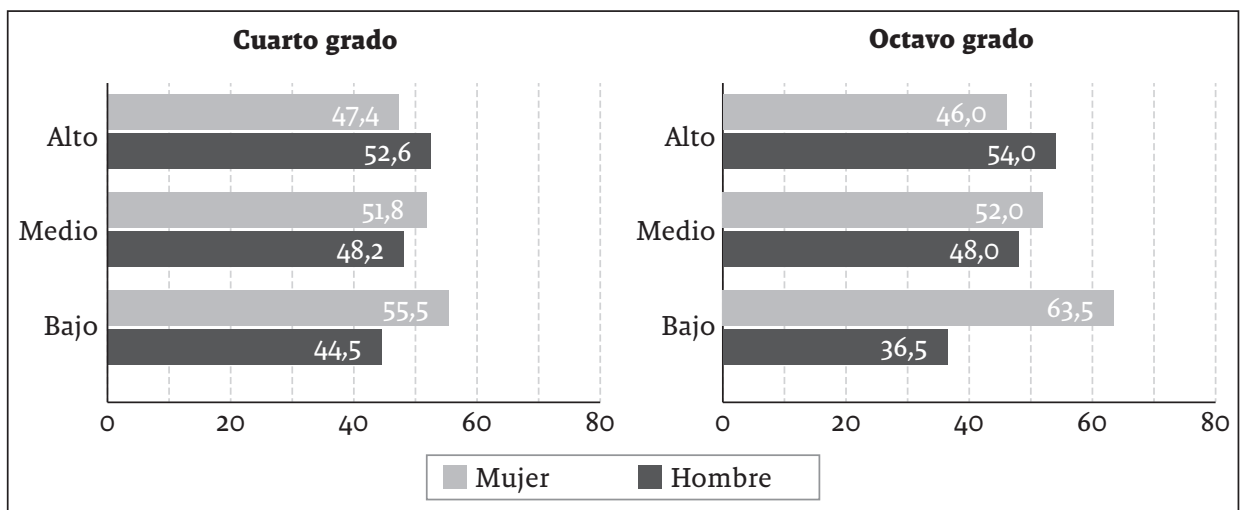

Fuente: Prueba TIMSS 2007. Cálculos del autor.

$\mathrm{Al}$ interactuar el género del estudiante con el sector del colegio, el comportamiento del índice de autoconfianza presenta tendencias a favor de los chicos, mayor proporción en el nivel alto y menor en el nivel bajo, tanto en los colegios oficiales como en los privados (gráfica 5). Una mirada a los puntos extremos deja ver que en el nivel alto, los chicos de los colegios privados, tanto de cuarto grado como de octavo, presentan en mayor proporción (un poco más de 13\%) creencias positivas acerca de su desempeño en matemáticas. En el otro extremo, en el nivel bajo, los porcentajes más altos corresponden a las chicas de los colegios 
oficiales, de cuarto y octavo grados, esto indica que la percepción negativa respecto al desempeño en matemáticas por parte de las chicas es $11,8 \%$ más alta en cuarto grado y se incrementa a $28,6 \%$ en octavo.

Gráfica 5. Proporción de estudiantes en cada nivel del índice de autoconfianza en el aprendizaje de las matemáticas según sector y género

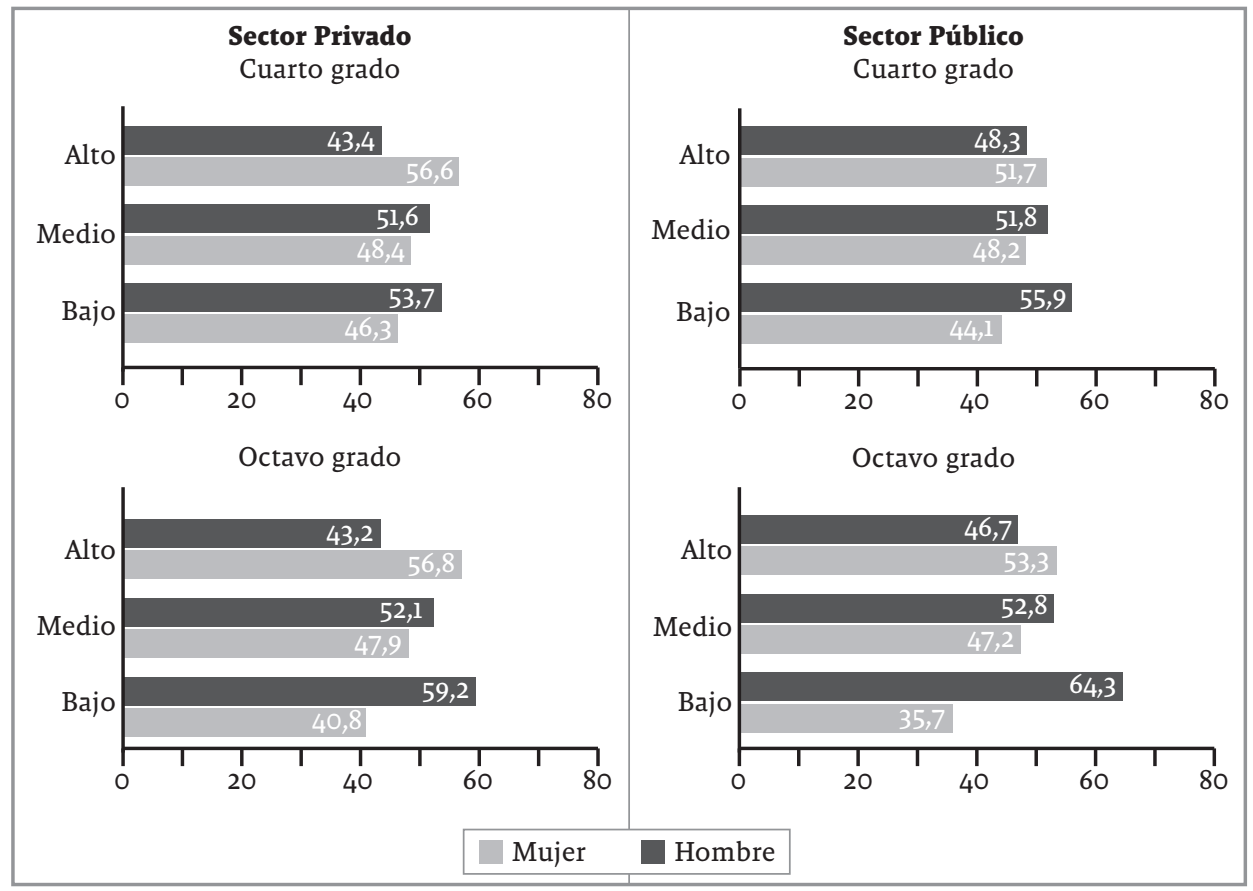

Fuente: Prueba TIMSS 2007. Cálculos del autor.

\subsection{Estimación del modelo}

La estimación del modelo jerárquico o multinivel descrito en la sección 4.2 se realiza bajo una estructura paso a paso; se inicia con la estimación del modelo más simple llamado modelo nulo, con el fin de calcular la variación total en cada una de las partes estocásticas del modelo y de evaluar la pertinencia del modelo en el análisis. Posteriormente se procede a incluir variables regresoras al modelo, evaluando así en cada etapa la capacidad explicativa de estas en los dos niveles del modelo.

El método de estimación utilizado en cada una de las etapas del modelo fue mínimos cuadrados generalizados iterados (IGLS por su notación en inglés), donde se tomó como variable dependiente el promedio de los cinco valores plausibles reportados en la prueba TIMSS 2007. Las estimaciones se realizan con la ayuda de la rutina runmlwin, propuesta por Leckie y Charlton (2013), la cual es aplicada al software Stata. Dado que se utilizó como variable dependiente el promedio de los cinco valores plausibles, los resultados obtenidos se compararon con los arrojados por la rutina $p v$ propuesta por Macdonald (2014) que trabaja simultáneamente con los cinco valores plausibles como variable dependiente. 
Los resultados de ambas estimaciones arrojaron leves diferencias tanto en los promedios estimados como en las varianzas.

Un análisis general de los modelos estimados (anexos 2 y 3) permite concluir que todos los signos corresponden a los esperados y planteados en las hipótesis de comportamiento, así mismo los coeficientes de pendiente como los de las varianzas en los diferentes modelos son altamente significativos. Solo en algunos casos, las interacciones entre variables reportan estimaciones no significativas, lo cual sugiere la inexistencia de diferencias entre los grupos de comparación.

Modelo 1: Modelo nulo, mide el rendimiento promedio global de los estudiantes de las diferentes escuelas. El coeficiente estimado en este modelo indica que, en promedio, los estudiantes de cuarto grado alcanzaron 360,1 puntos en la prueba de matemáticas, con un error estándar de 69 puntos, por tanto, el intervalo de confianza de $95 \%$ permite ver que la variación del rendimiento entre las escuelas toma valores entre $(241,3 ; 479,0)=(360,1 \pm 1,96 * 60,6)$, donde $60,6=\sqrt{ } 3.677,5$ es desviación estándar de los estudiantes en las escuelas. El intervalo deja ver que solo unos pocos estudiantes superan el valor umbral de 475 puntos en la cola superior. El modelo confirma los resultados del análisis descriptivo donde se indicó que $89 \%$ de los estudiantes alcanzaron resultados por debajo del nivel 3 cuyo umbral es 475 puntos según la clasificación TIMSS.

En octavo grado, el desempeño promedio en las escuelas fue de 378,3 puntos con un error estándar de 88 puntos; la variación aleatoria de las respuestas en la prueba entre las escuelas fue de $51,3=\sqrt{2} .632,8$ puntos, por tanto, el rango en el que varían los desempeños es $(277,8 ; 478,8)=\left(378,3 \pm 1,96^{*} 51,3\right)$, con una confianza del $95 \%$. El intervalo muestra que aunque haya aumentado el desempeño promedio, el valor umbral para alcanzar el nivel 3 de 475 puntos está muy próximo al límite superior del intervalo. Similar a los resultados de cuarto grado, $95 \%$ de los estudiantes ubicados alrededor de la media presentan rendimientos muy similares tanto en cuarto como en octavo; solo 2,5\% de las respuestas en la cola superior alcanzan un desempeño comparable con los países desarrollados.

El índice de correlación intraclase (ICC) que mide la proporción de variación debida a los colegios o la similaridad entre los estudiantes de los mismos colegios, indica que 50\% de la variación en el desempeño de los estudiantes en cuarto grado y $46,7 \%$ en octavo se debe a las altas diferencias entre los colegios; este es un indicativo de la marcada segregación del sistema educativo colombiano. El paso a seguir es tratar de explicar esta proporción de varianza a partir de la inclusión de variables en ambos niveles del modelo, estudiante como colegio.

Modelo 2: se obtiene luego de incluir la variable género, el coeficiente asociado mide el desempeño diferencial entre chicos y chicas. El modelo predice que en cuarto grado los chicos obtuvieron 14,5 puntos adicionales en el rendimiento promedio respecto a las niñas, mientras que en octavo, la brecha, igualmente a favor de los chicos, se duplicó alcanzando 28,4 puntos en promedio. Es de resaltar la capacidad explicativa del género de la variabilidad de los desempeños en ambos niveles del modelo estimado, tanto en cuarto grado como en octavo, no obstante, el índice de correlación intraclase aumenta. El aporte de la variable género a la explicación de la varianza en el primer nivel fue de $1,4 \%$ en cuarto grado y de $5,8 \%$ en octavo. 
Modelo 3: mide el efecto diferencial en el desempeño según el sector al que pertenece el colegio. Los resultados muestran que los estudiantes de las instituciones privadas, en promedio, obtuvieron desempeños más destacados que los de los colegios públicos. En cuarto grado la brecha alcanzó 75 puntos, mientras que en octavo el diferencial promedio fue de 63 puntos. La variable sector explica ampliamente la variación en los puntajes en el segundo nivel del modelo, situación que se refleja en la disminución que presenta el ICC al caer 5 puntos; comportamiento que se presenta en ambos grados.

Modelo 4: la especificación de este modelo se obtiene a partir de las variables: índice de nivel socioeconómico (ISE) del estudiante, el género y sector del colegio. En primer lugar, se observa que el efecto diferencial de las variables de género y sector no presentó variaciones significativas respecto a los dos modelos antes explicados. Similar a lo encontrado en estudios para otros países, el ISE tiene un efecto positivo sobre el desempeño académico del estudiante. El modelo indica que un aumento de una unidad en el ISE le representa al estudiante 10,5 puntos adicionales en el desempeño en matemáticas si es de cuarto grado y 7,7 puntos si es de octavo. Dos aspectos a resaltar de estos resultados son: primero, el impacto del ISE sobre los logros del estudiante es más importante al inicio del ciclo escolar que en los grados siguientes, por lo que el proceso de formación de habilidades que se da en los primeros años de requiere de inversiones más altas. Segundo, como lo muestran muchos estudios, el ISE es importante para explicar los logros de los estudiantes, pero existen otros factores que explican en mayor proporción el desempeño.

El ISE explica la variabilidad de los errores en una baja proporción en la ecuación del nivel 2 (ecuación de colegios) en ambos grados, cuarto y octavo; muestra de esto es la leve caída en el ICC respecto al modelo inmediatamente anterior (modelo 3).

Modelo 5: una medida del efecto de los compañeros de clase del estudiante $i$ (ECC) se adiciona a la especificación anterior; esta variable pretende medir la influencia de los compañeros de la clase en las escuelas sobre el desempeño del estudiante $i$. El modelo predice, manteniendo la demás información constante, que un incremento en una unidad en el ECC produce un aumento en el desempeño medio en la prueba de matemáticas de 193,4 puntos si el estudiante es de cuarto grado, y de 105,0 si es de octavo. Es de anotar que la interacción de la variable ECC con la variable de género no es significativa, esto indica, como era de esperarse, que el efecto de los pares es idéntico si el estudiante es niño o niña. La variable ECC se puede considerar como una medida del grado de segregación del sistema educativo, similar a lo encontrado por Duarte y Bos (2012). Los resultados indican que los alumnos de condiciones socioeconómicas altas tienen compañeros de similares condiciones, lo que incide de manera positiva en el rendimiento académico. Por el contrario, los alumnos de bajo nivel socioeconómico que, asisten a escuelas públicas con pares de idéntica condición social y económica, alcanzan desempeños promedio igualmente bajos, producto de la influencia del grupo al que pertenecen; efectos endógenos que perpetúan de esta manera las condiciones de segregación social y económica.

La proporción de variación entre escuelas explicada por el índice ECC tomando como referencia el modelo inmediatamente anterior es cercana a $10 \%$ en el 
modelo de cuarto grado y a $12 \%$ en el de octavo, indicando lo desigual que es la educación colombiana. Es de notar que la caída en la varianza del error del nivel 1 en ambos modelos - cuarto y octavo- es mínima.

Modelo 6: en este modelo se agrega una variable que mide el nivel de autoconfianza de los estudiantes en el aprendizaje de las matemáticas, para su modelación se construyen seis variables producto de la interacción entre las variables género (hombre y mujer) y el nivel de autoconfianza (alto, medio y bajo); para el análisis se deja como variable de referencia las mujeres con bajo nivel de autoconfianza.

En los grados cuarto y octavo, el efecto diferencial respecto a la categoría de referencia (chicas con bajo nivel de autoconfianza) es positivo; esto indica que a medida que aumenta la autoconfianza en el aprendizaje, tanto en los chicos como en las chicas, el desempeño en matemáticas mejora significativamente. En cuarto grado se observa que respecto al bajo nivel de autoconfianza, cuando se tiene un nivel medio de creencias positivas, el desempeño aumenta 24,4 puntos si es una chica y solo 20,1 puntos si es chico; pero si el nivel de autoconfianza es alto, en promedio las chicas alcanzan 56,4 puntos adicionales en el desempeño y los chicos 60,5.

En octavo las brechas son más amplias, tanto al comparar con la categoría de referencia como entre chicos y chicas. En promedio, las chicas con nivel medio de autoconfianza alcanzaron en la prueba un diferencial de 17,05 puntos, y las que tienen un alto nivel de autoconfianza 54,58 puntos. Los chicos con bajo nivel de autoconfianza obtuvieron en promedio 38,7 puntos adicionales, 53,4 si se ubican en el nivel medio y de 92,3 si presentan un alto nivel.

La variable de autoconfianza en el aprendizaje de las matemáticas explica ampliamente las variaciones en el desempeño en la ecuación del estudiante; en cuarto grado la proporción de variación explicada es superior a $13 \%$, aumentando en octavo a 17,5\%; no obstante, esta capacidad explicativa no se refleja en el ICC, cuya disminución fue muy leve.

\section{Conclusiones}

Los resultados permiten concluir que independiente del nivel de formación alcanzada por los estudiantes — grados cuarto u octavo- el nivel socioeconómico del hogar del estudiante, aunque importante al momento de explicar las diferencias en el desempeño académico, per se no es la característica de mayor influencia; pero, sí tiene una alta influencia en los mecanismos de formación de habilidades de los alumnos, en la elaboración de códigos y formas del discurso más elaborado y en la adquisición de capital cultural, factores que son determinantes para alcanzar altos desempeños promedio.

Lo anterior explica, en parte, las amplias diferencias en el desempeño a favor de los colegios privados, situación que se pone en evidencia al incluir la medición del efecto de los pares. El principal hallazgo obtenido a partir de este resultado es la amplia segmentación del sistema educativo colombiano; los estudiantes de alto nivel socioeconómico tienen compañeros de similares condiciones y asisten a colegios privados, generando efectos correlacionados que influyen de manera positiva sobre el rendimiento académico. Por su parte, aquellos con 
niveles socioeconómicos bajos asisten a colegios públicos con pares de idéntica condición social y económica, obteniendo en promedio logros igualmente bajos producto de una mezcla entre efectos endógenos - predomina el desempeño promedio del grupo de referencia-y efectos exógenos; sobre el desempeño influyen igualmente las escasas condiciones socioeconómicas. En conclusión, en Colombia la educación varía según la condición social de los estudiantes; resultado que se observa tanto en el análisis descriptivo como a partir de los modelos estimados.

Los resultados dejan ver, en primer lugar una amplia brecha en el desempeño en la prueba de matemáticas según tipo de colegio, tanto en cuarto como en octavo grados; las amplias diferencias en el desarrollo de las habilidades cognitivas entre los alumnos de alto y bajo nivel socioeconómico - más que en las condiciones innatas - son la principal causa, pero hay otros factores que inciden también relacionados con el entorno familiar. Las creencias o percepciones que el estudiante tiene respecto a la capacidad de desempeñarse en matemáticas, así como la motivación y el esfuerzo se construyen a lo largo del tiempo, pero sus inicios se presentan en el hogar, de ahí que la formación de las creencias en sí mismo esté en primera instancia influenciada por este.

La autoconfianza del estudiante en su capacidad de aprendizaje, no solo en el área de las matemáticas sino en cualquiera otra área del conocimiento, es el factor fundamental que se requiere para llegar a alcanzar logros que sean competitivos más aún en un ambiente internacional. Los datos muestran que está condición es la principal causante de las brechas entre hombres y mujeres, en especial los de los colegios públicos.

La prueba TIMSS deja ver que, tanto en cuarto como en octavo grados, las mujeres son quienes presentan los niveles más bajos de autoconfianza en el desempeño en matemáticas; lo preocupante es que la formación de creencias es un proceso dinámico que aumenta o disminuye según el desempeño del estudiante. El análisis descriptivo muestra que a medida que avanza el proceso de formación (comparando cuarto y octavo grados) las mujeres con bajos niveles de autoconfianza consolidan sus creencias sobre sí mismas, es decir, se ratifican en que ellas no son buenas para las matemáticas, con la consecuente disminución en su motivación por aprender matemáticas y menores niveles de esfuerzo.

En cuanto a la política, el estudio deja en evidencia la necesidad de impulsar procesos de intervención por parte del ente regulador que controlen de manera diferenciada las condiciones sociales y económicas del estudiante. La intervención debe procurar que los niños de bajo nivel socioeconómico inicien procesos de formación de habilidades a edades tempranas con el fin de disminuir brechas cognitivas y, especialmente, no cognitivas.

\section{Referencias bibliográficas}

Arnott, Richard y John Rowse. «Peer Group Effects and Educational Attainment». Journal of Public Economics, $\mathrm{n}^{\circ}$ 32, 1987: 287-305.

Arrow, Kenneth. «Higher Education as a Filter». Journal of Public Economics, Vol. 2, $\mathrm{n}^{\circ}$ 3, 1973: 193-216.

Bandura, Albert. «Perceived Self-Efficacy in Cognitive Development and Functioning». Educational Psychologist, Vol. 28, nº 2, 1993: 117-148. 
Bandura, Albert. «Self-Efficacy - Toward a Unifying Theory of Behavioral Change». Psychological Review, Vol. 84, nº 2, 1977: 191-215.

Bandura, Albert. «Self-Efficacy Mechanism in Human Agency». American Psychologist, Vol. 37, $\mathrm{n}^{\circ}$ 2, 1982: 122-147.

Becker, Gary. Human Capital: A Theoretical and Empirical Analysis, with Special Reference to Education. Chicago: University of Chicago Press, [1964] 1994.

Bernstein, Basil. Class, Codes and Control, Theoretical Studies Towards a Sociology of Language. Vol. 1. Londres: Paladin, 1975.

Bourdieu, Pierre. Poder, derecho y clases sociales, $2^{\circ}$ Edición. España: Descleé de Brouwer, 2000.

Bourdieu, Pierre y Jean Passeron. Los estudiantes y la cultura. Argentina: Editorial Labor, 1973.

Card, David. «The Causal Effect of Education on Earnings». En Handbook of Labor Economics, Vol. 3A, de Orley Ashenfelter y David Card, 1802-1859. Amsterdam: Elsevier, 1999.

Coleman, James y otros. Equality of Educational Opportunity. Washington, D.C.: U.S. Government Printing Office, 1966.

Cunha, Flavio y James Heckman. «The Technology of Skill Formation». American Economics Review, Vol. 97, $\mathrm{n}^{\circ}$ 2, 2007: 31-47.

Duarte, Jesús y María Bos. «Desigualdad en los aprendizajes de los alumnos colombianos». BID, ${ }^{0} 17$ (julio 2012): 1-8.

Ellison, Glenn y Ashley Swanson. «The Gender Gap in Secondary School Mathematics at High Achievement Levels: Evidence from the American Mathematics Competitions». Journal of Economic Perspectives, Vol. 24, $\mathrm{n}^{\circ}$ 2, 2010: 109-128.

Garner, Catherine y Stephen Raudenbush. «Neighborhood Effects on Educational Attainment: A Multilevel Analysis». Sociology of Education, Vol. 64, $\mathrm{n}^{\circ}$ 4, 1991: 251-262.

Gaviria, Alejandro y Raphael Steven. «School-Based Peer Effects and Juvenile Behavion». The Review of Economics and Statistics, Vol. 83, n 2, 2001: 257-268.

Giddens, Anthony. Sociología. $4^{\circ}$ Edición. España: Alianza Editorial, 2001.

Heckman, James, Jora Stixrud y Sergio Urzua. «The Effects of Cognitive and Noncognitive Abilities on Labor Market Outcomes and Social Behavior». NBER, $n^{\circ} 12.006$, 2006: 1-80.

ICFES. Análisis de las diferencias de género en el desempeño de estudiantes colombianos en matemáticas y lenguaje. Bogotá, D.C.: Icfes, 2013.

ICFES. Resultados de Colombia en TIMSS 2007 - Resumen ejecutivo. Informe Resultados Internacionales. Bogotá: ICFES, 2010.

Illich, Ivan. La sociedad desescolarizada. Barcelona: Barral Editores, 1974.

Leckie, George y Chris Charlton. «runmlwin: A Program to Run the MLwiN Multilevel Modelling Software from within Stata». Journal of Statistical Software, Vol. 52, $\mathrm{n}^{\circ} 11,2013: 1-40$.

Longford, Nick y Murray Aikkin. «Statistical Modelling Issues in School Effectiveness Studies». Journal of the Royal Statistical Society, Series A, $n^{\circ}$ 149, 1986: 1-43.

Macdonald, Kevin. PV: Stata Module to Perform Estimation with Plausible Values, 2014. http://EconPapers.repec.org/RePEc:boc:bocode:\$456951 (último acceso: 26 de junio de 2014). 
Manski, Charles. «Identification of Endogenous Social Effects: The Reflection Problem». The Review of Economic Studies, Vol. 60, nº 3, 1993: 531-542.

Mullis, Ina, Michael Martin y Pierre Foy. TIMSS 2007 International Mathematics Report. Chestnut Hill, MA: TIMSS \& PIRLS International Study Center, Boston College, 2008.

Niederle, Muriel y Lise Vesterlund. «Explaining the Gender Gap in Math Test Scores: The Role of Competition». The Journal of Economic Perspectives, Vol. 24, nº 2, 2010 : 129-144.

Pajares, Frank. «Self-Efficacy Beliefs in Academic Settings». Review of Educational Research, Vol. 66, $\mathrm{n}^{\circ}$ 4, 1996: 543-578.

Reardon, Sean. «The Widening Academic Achievement Gap between the Rich and the Poor». Community Investments, Vol. 24, $\mathrm{n}^{\circ}$ 2, 2012: 19-39.

Schultz, Theodore. «Investment in Human Capital». The American Economic Review, Vol. 51, no 1, 1961: 1-17.

Spelke, Elizabeth. «Sex Differences in Intrinsic Aptitude for Mathematics and Science? A Critical Review». American Psychologist, Vol. 60, nº 9, 2005: 950-958.

Spence, Michael. «Job Market Signaling». The Quarterly Journal of Economics, Vol. 87, $\mathrm{n}^{\circ}$ 3, 1973: 355-374.

Stiglitz, Joseph. «The Theory of Screening, Education and the Distribution of Income». The American Economic Review, Vol. 65, nº 3, 1975: 283-300.

Vivas, Harvy. 2008. Educación, Background familiar y calidad de los entornos locales en Colombia. Tesis Doctoral, Departament d'Economia Aplicada, Universitat Autònoma de Barcelona-UAB.

Vivas, Harvy, Juan Correa y Jorge Domínguez. «Potencial de logro educativo, entorno socioeconómico y familiar: una aplicación empírica con variables latentes». Sociedad y economía, $\mathrm{n}^{\circ}$ 21, 2011: 99-124.

\section{Anexos}

Anexo 1. Índice de autoconfianza en el aprendizaje de las matemáticas según sexo. Proporción de estudiantes en cada nivel

\begin{tabular}{|c|c|c|c|c|c|c|c|c|}
\hline \multirow{2}{*}{ Sexo } & \multicolumn{4}{|c|}{ Cuarto grado } & \multicolumn{4}{|c|}{ Octavo grado } \\
\hline & Alto & Medio & Bajo & Total & Alto & Medio & Bajo & Total \\
\hline \multirow{3}{*}{ Mujer } & $1.059,0$ & 950,0 & 167,0 & $2.176,0$ & $1.024,0$ & 978,0 & 397,0 & $2.399,0$ \\
\hline & $48,7 \%$ & $43,7 \%$ & $7,7 \%$ & $100,0 \%$ & $42,7 \%$ & $40,8 \%$ & $16,6 \%$ & $100,0 \%$ \\
\hline & $47,4 \%$ & $51,8 \%$ & $55,5 \%$ & $49,8 \%$ & $46,0 \%$ & $52,0 \%$ & $63,5 \%$ & $50,7 \%$ \\
\hline \multirow{3}{*}{ Hombre } & $1.177,0$ & 884,0 & 134,0 & $2.195,0$ & $1.202,0$ & 902,0 & 228,0 & $2.332,0$ \\
\hline & $53,6 \%$ & $40,3 \%$ & $6,1 \%$ & $100,0 \%$ & $51,5 \%$ & $38,7 \%$ & $9,8 \%$ & $100,0 \%$ \\
\hline & $52,6 \%$ & $48,2 \%$ & $44,5 \%$ & $50,2 \%$ & $54,0 \%$ & $48,0 \%$ & $36,5 \%$ & $49,3 \%$ \\
\hline \multirow{3}{*}{ Total } & $2.236,0$ & $1.834,0$ & 301,0 & $4.371,0$ & $2.226,0$ & $1.880,0$ & 625,0 & $4.731,0$ \\
\hline & $51,2 \%$ & $42,0 \%$ & $6,9 \%$ & $100,0 \%$ & $47,1 \%$ & $39,7 \%$ & $13,2 \%$ & $100,0 \%$ \\
\hline & $100,0 \%$ & $100,0 \%$ & $100,0 \%$ & $100,0 \%$ & $100,0 \%$ & $100,0 \%$ & $100,0 \%$ & $100,0 \%$ \\
\hline
\end{tabular}

Fuente: Prueba TIMSS 2007. Cálculos del autor 
Anexo 2. Estimación modelo nulo y extensiones cuarto grado. TIMSS 2007

\begin{tabular}{|c|c|c|c|c|c|c|}
\hline Variables & Modelo I & Modelo 2 & Modelo 3 & Modelo 4 & Modelo 5 & Modelo 6 \\
\hline \multicolumn{7}{|c|}{ FP1: Efectos marginales del nivel 1} \\
\hline \multirow{2}{*}{ Constante } & 360,12 & 352,72 & 349,93 & 343,65 & 357,89 & 323,3 \\
\hline & $(0,000)^{*}$ & $(0,000)^{*}$ & $(0,000)^{*}$ & $(0,000)^{*}$ & $(0,000)^{*}$ & $(0,000)^{*}$ \\
\hline \multirow{2}{*}{ Género: hombre } & & 14,48 & & 14,19 & 14,25 & 11,46 \\
\hline & & $(0,000)^{*}$ & & $(0,000)^{*}$ & $(0,000)^{*}$ & $(0,004)^{*}$ \\
\hline \multirow{2}{*}{ Sector: privado } & & & 74,42 & 70,28 & 9,05 & 8,22 \\
\hline & & & $(0,000)^{*}$ & $(0,000)^{*}$ & $(0,420)^{*}$ & $(0,451)^{*}$ \\
\hline \multirow{2}{*}{$\begin{array}{l}\text { Índice de nivel } \\
\text { socioeconómico }\end{array}$} & & & & 10,53 & 11,74 & 4,83 \\
\hline & & & & $(0,006)^{*}$ & $(0,002)^{*}$ & $(0,174)^{*}$ \\
\hline \multirow{2}{*}{ Efecto de los pares } & & & & & 193,44 & 184,09 \\
\hline & & & & & $(0,000)^{*}$ & $(0,000)^{*}$ \\
\hline \multirow{2}{*}{$\begin{array}{c}\text { Mujer con } \\
\text { autoconfianza media }\end{array}$} & & & & & & 24,41 \\
\hline & & & & & & $(0,000)^{*}$ \\
\hline \multirow{2}{*}{$\begin{array}{c}\text { Mujer con } \\
\text { autoconfianza alta }\end{array}$} & & & & & & 56,64 \\
\hline & & & & & & $(0,000)^{*}$ \\
\hline \multirow{2}{*}{$\begin{array}{c}\text { Hombre con } \\
\text { autoconfianza baja }\end{array}$} & & & & & & 9,39 \\
\hline & & & & & & $(0,116)^{*}$ \\
\hline \multirow{2}{*}{$\begin{array}{c}\text { Hombre con } \\
\text { autoconfianza media }\end{array}$} & & & & & & 20,11 \\
\hline & & & & & & $(0,000)^{*}$ \\
\hline \multirow{2}{*}{$\begin{array}{c}\text { Hombre con } \\
\text { autoconfianza alta }\end{array}$} & & & & & & 60,51 \\
\hline & & & & & & $(0,000)^{*}$ \\
\hline \multicolumn{7}{|c|}{ RP2: Efectos aleatorios nivel 2} \\
\hline \multirow{2}{*}{ Varianza (constante) } & $3.677,51$ & $3.668,29$ & $3.015,01$ & $2.917,65$ & $1.971,73$ & $1.708,22$ \\
\hline & $(0,000)^{*}$ & $(0,000)^{*}$ & $(0,000)^{*}$ & $(0,000)^{*}$ & $(0,000)^{*}$ & $(0,000)^{*}$ \\
\hline \multicolumn{7}{|c|}{ RPı: Efectos aleatorios nivel 1} \\
\hline & $3.684,33$ & $3.633,66$ & $3.684,49$ & $3.630,86$ & $3.634,65$ & 3.183 \\
\hline & $(0,000)^{*}$ & $(0,000)^{*}$ & $(0,000)^{*}$ & $(0,000)^{*}$ & $(0,000)^{*}$ & $(0,000)^{*}$ \\
\hline \multicolumn{7}{|l|}{ Estadísticos } \\
\hline & $-26.925,3$ & $-26.892,7$ & $-26.912,2$ & $-26.875,6$ & $-26.852,3$ & $-26.531,1$ \\
\hline & $50,0 \%$ & $50,2 \%$ & $45,0 \%$ & $44,6 \%$ & $35,2 \%$ & $34,9 \%$ \\
\hline & $53.850,68$ & $53.785,31$ & $53.824,35$ & $53.751,11$ & $53.704,66$ & $53.062,25$ \\
\hline & $53.876,13$ & $53.819,24$ & $53.858,28$ & 53.802 & $53.764,04$ & $53.164,04$ \\
\hline & 4.831 & 4.831 & 4.831 & 4.831 & 4.831 & 4.831 \\
\hline
\end{tabular}

* Valores de probabilidad entre paréntesis.

Fuente: Prueba TIMSS 2007. Cálculos del autor. 
Anexo 3. Estimaciones modelo nulo y extensiones octavo grado. TIMSS 2007

\begin{tabular}{|c|c|c|c|c|c|c|}
\hline Variables & Modelo 1 & Modelo 2 & Modelo 3 & Modelo 4 & Modelo 5 & Modelo 6 \\
\hline \multicolumn{7}{|c|}{ FP1: Efectos marginales del nivel 1} \\
\hline \multirow{2}{*}{ Constante } & 378,3 & 364,2 & 367,3 & 355,1 & 370,9 & 341,23 \\
\hline & $(0,000)^{*}$ & $(0,000)^{*}$ & $(0,000)^{*}$ & $(0,000)^{*}$ & $(0,000)^{*}$ & $(0,000)^{*}$ \\
\hline \multirow{2}{*}{ Género: hombre } & & 28,4 & & 27,6 & 27,6 & $-13,06$ \\
\hline & & $(0,000)^{*}$ & & $(0,000)^{*}$ & $(0,000)^{*}$ & $(0,089)^{*}$ \\
\hline \multirow{2}{*}{ Sector: privado } & & & 62,3 & 56,1 & $-12,6$ & $-13,21$ \\
\hline & & & $(0,000)^{*}$ & $(0,000)^{*}$ & $(0,219)^{*}$ & $(0,2)^{*}$ \\
\hline \multirow{2}{*}{$\begin{array}{l}\text { Índice de nivel } \\
\text { socioeconómico }\end{array}$} & & & & 7,7 & 9,2 & 5,38 \\
\hline & & & & $(0,006)^{*}$ & $(0,002)^{*}$ & $(0,000)^{*}$ \\
\hline \multirow{2}{*}{ Efecto de los pares } & & & & & 105,0 & 103,11 \\
\hline & & & & & $(0,000)^{*}$ & $(0,000)^{*}$ \\
\hline \multirow{2}{*}{$\begin{array}{c}\text { Mujer con } \\
\text { autoconfianza media }\end{array}$} & & & & & & 17,05 \\
\hline & & & & & & $(0,000)^{*}$ \\
\hline \multirow{2}{*}{$\begin{array}{l}\text { Mujer con } \\
\text { autoconfianza alta }\end{array}$} & & & & & & 54,58 \\
\hline & & & & & & $(0,000)^{*}$ \\
\hline \multirow{2}{*}{$\begin{array}{c}\text { Hombre con } \\
\text { autoconfianza baja }\end{array}$} & & & & & & 38,71 \\
\hline & & & & & & $(0,000)^{*}$ \\
\hline \multirow{2}{*}{$\begin{array}{l}\text { Hombre con } \\
\text { autoconfianza media }\end{array}$} & & & & & & 53,44 \\
\hline & & & & & & $(0,000)^{*}$ \\
\hline \multirow{2}{*}{$\begin{array}{c}\text { Hombre con } \\
\text { autoconfianza alta }\end{array}$} & & & & & & 92,34 \\
\hline & & & & & & $(0,000)^{*}$ \\
\hline \multicolumn{7}{|c|}{ RP2: Efectos aleatorios nivel 2} \\
\hline \multirow{2}{*}{ Varianza (constante) } & $2.632,84$ & $2.552,74$ & $2.070,53$ & $1.886,18$ & $1.080,05$ & $1.020,25$ \\
\hline & $(0,000)^{*}$ & $(0,000)^{*}$ & $(0,000)^{*}$ & $(0,000)^{*}$ & $(0,000)^{*}$ & $(0,000)^{*}$ \\
\hline \multicolumn{7}{|l|}{$\mathrm{RP} 1$} \\
\hline \multirow{2}{*}{ Varianza (constante) } & $3.006,27$ & $2.831,79$ & $3.006,25$ & $2.825,11$ & $2.824,77$ & $2.331,14$ \\
\hline & $(0,000)^{*}$ & $(0,000)^{*}$ & $(0,000)^{*}$ & $(0,000)^{*}$ & $(0,000)^{*}$ & $(0,000)^{*}$ \\
\hline \multicolumn{7}{|l|}{ Estadísticos } \\
\hline Función de verosimilitud & $-27.000,0$ & $-27.000,0$ & $-27.000,0$ & $-27.000,0$ & $-26.000,0$ & $-26.007,6$ \\
\hline $\begin{array}{l}\text { Indice de correlación } \\
\text { intraclase (ICC) }\end{array}$ & $46,7 \%$ & $47,4 \%$ & $40,8 \%$ & $40,0 \%$ & $27,7 \%$ & $30,4 \%$ \\
\hline Deviance & $53.352,13$ & $53.064,9$ & $53.317,98$ & $53.010,76$ & $52.932,71$ & $52.015,28$ \\
\hline Criterio de Schuartz & $53.377,61$ & $53.098,86$ & $53.351,95$ & $53.061,71$ & $52.992,15$ & $52.117,17$ \\
\hline Nro. de observaciones & 4.873 & 4.873 & 4.873 & 4.873 & 4.873 & 4.873 \\
\hline
\end{tabular}

* Valores de probabilidad entre paréntesis.

Fuente: Prueba TIMSS 2007. Cálculos del autor. 